IV. Materials

Abstract

I. Introduction

II. Rationale

III. Methods

V. Discussion

VI. Summary

References

\title{
Nondenaturing Electrophoresis as a Tool to Investigate Tubulin Complexes
}

\author{
Mónica López Fanarraga ${ }^{\star}$, Gerardo Carranza ${ }^{\star}$, Raquel \\ Castaño $^{\star}$, Sofia Nolasco ${ }^{\star},{ }^{\dagger}$, J. Avila ${ }^{\star}$, and J.C. Zabala ${ }^{\star}$ \\ *Departamento de Biología Molecular, Facultad de Medicina, IFIMAV-Universidad de Cantabria, \\ Santander, Spain \\ †Instituto Gulbenkian de Ciência, 2781-901 Oeiras, Portugal \\ †Centro de Biología Molecular (CSIC-UAM), Universidad Autónoma de Madrid, 28049 Cantoblanco, \\ Madrid, Spain
}

A. Native Gels and Western Blotting

B. Analysis of Purified Tubulin: Tubulin Stability Assessed Using Nondenaturing Gels

C. Kinetic Analysis of Tubulin Complexes and Characterization of In VitroSynthesized Products

D. Isotype-Specific Antibody Analysis

E. 2-D Native SDS Electrophoresis

F. Preparation and Analysis of Subtilisin-Treated Dimers

G. Other Applications of Nondenaturing Electrophoresis: Stability Study of the In Vivo Tubulin Complexes by Native Electrophoresis

Acknowledgments 


\section{Abstract}

A protein molecule may exist as a monomer, homo-oligomer, or hetero-oligomer in a multiprotein complex. One-dimensional (1-D) native electrophoresis has long been used to characterize tubulins and their complexes. In this chapter, we describe the simplest way to identify the state of aggregation of commercial or homemade tubulins for further studies based on 1-D electrophoresis under nondenaturing conditions. We present a series of detailed protocols that can be used to analyze the maturation of $\alpha$ - and $\beta$-tubulins and to identify the complexes formed during the folding and dimerization pathway as well as their stability.

\section{Introduction}

From the pioneering work of Smithies (1955) and Markert and Hunter (1959) followed by the descriptions of disk electrophoresis by Ornstein and Davis, both in 1964, electrophoresis has become one of the most effective methods for separating single macromolecules or complexes of several macromolecules according to the size and charge of the different molecular entities. To isolate single proteins, Shapiro et al. (1967) reported the use of polyacrylamide electrophoresis in the presence of the anionic detergent sodium dodecyl sulfate (SDS). In this condition, single polypeptide chains can be isolated because their fractionation depends only on the molecular weight of the polypeptide chains. These results were later confirmed by Weber and Osborn (1969), who showed the reliability of molecular weight determinations by dodecyl sulfate polyacrylamide gel electrophoresis (PAGE). This technique was refined by Laemmli (1970). Although this excellent method for protein fractionation was described mainly for the characterization of single proteins present in isolated fractions, addition of the denaturing detergent SDS makes it impossible to determine whether the proteins present interact with their neighbors or with themselves by forming multimolecular complexes of physiological relevance. Thus, alternative techniques without denaturing agents were tested with the aim of isolating and characterizing monomers, dimers, and multimolecular complexes of a protein and its associated proteins. Zabala and Cowan (1992) described a technique for the fractionation of monomers, dimers, and multimolecular complexes of tubulin using nondenaturing conditions. This widely used technique allows one to perform functional studies on the assembly of multimolecular protein complexes. In this chapter, we describe different applications of nondenaturing gel electrophoresis to examine the structure of tubulin complexes.

\section{Rationale}

Nondenaturing gel electrophoresis is used to separate proteins by retaining their higher-order structure and their interactions with other polypeptides; this is achieved by avoiding the use of denaturing agents in the gel. Unlike SDS-PAGE, in nondenaturing gel electrophoresis, the migration of proteins depends on their global shape and charge, implying that the mobility of a protein complex can vary 
according to the nature of its conformation. In this kind of approach, one must remember that the electric charge of a protein, or a protein complex, depends on the composition of the electrophoresis buffer.

Although nondenaturing electrophoresis provides less resolution than does SDSPAGE, this technique is useful because it permits the study of protein-protein and protein-ligand interactions. Native gel electrophoresis is used widely in many types of studies such as the analysis of the charge changes caused by chemical degradation, changes between the folded and unfolded conformation, formation of oligomers and aggregates, identification of individual proteins within a complex, characterization of nucleoprotein particles, and protein-protein- or protein-ligand-binding events. Native gel electrophoresis is also an excellent tool for analyzing the stability of samples, the effects of excipients, and antibody-protein complexes (Safer, 1994).

The combination of native electrophoresis and Western blotting is a powerful tool to identify the individual protein components in a complex by immunodetection. In this type of combined method, it is important to pay particular attention to negative results because the epitopes recognized by the antibodies can be unavailable in the protein complex structure. In addition, in those cases where more precise proteomic analysis of the components should be performed, nondenaturing gel electrophoresis can be complemented by a second SDS-PAGE dimension.

Two main types of native gels are used in protein electrophoresis: polyacrylamide and agarose. The concentrations of acrylamide and bisacrylamide determine the resolution range of the gel. Agarose gels are used for electrophoresis of proteins generally larger than $200 \mathrm{kDa}$.

We have developed a protocol that can be used to routinely investigate different aspects of our main research subject, tubulin, and its folding intermediates (Fig. 1). Here we describe several examples of native PAGE applications that we currently use in our studies. These include the quality analysis of purified tubulin, characterization of properly folded tubulin dimers, analysis of tubulin complex stability and assembly kinetics, and characterization of the in vitro-synthesized tubulin complexes. Two additional applications of the technique are also possible: the analysis of single protein components in two-dimensional (2-D) native SDS electrophoresis and analysis of the efficiency of the preparation of subtilisin-treated tubulin dimers; these additional applications show the versatility of this technique.

\section{Methods}

\section{A. Native Gels and Western Blotting}

Many techniques based on native electrophoresis have been developed to separate native proteins and protein complexes. Among them, blue native electrophoresis is one of the best known techniques (Wittig and Schägger, 2008; Burré et al., 2009). This technique was developed initially to study membrane proteins, although it is now used more widely. Most of the native electrophoresis systems use acrylamidegradient gels. In our system, we use a native continuous-buffer system, which allows the addition of glycerol or nonionic detergents in the buffer, although we typically do not use them. We routinely do not degas the polyacrylamide mix, but this can be done in a sidearm flask under vacuum to speed the polymerization. To prepare the 


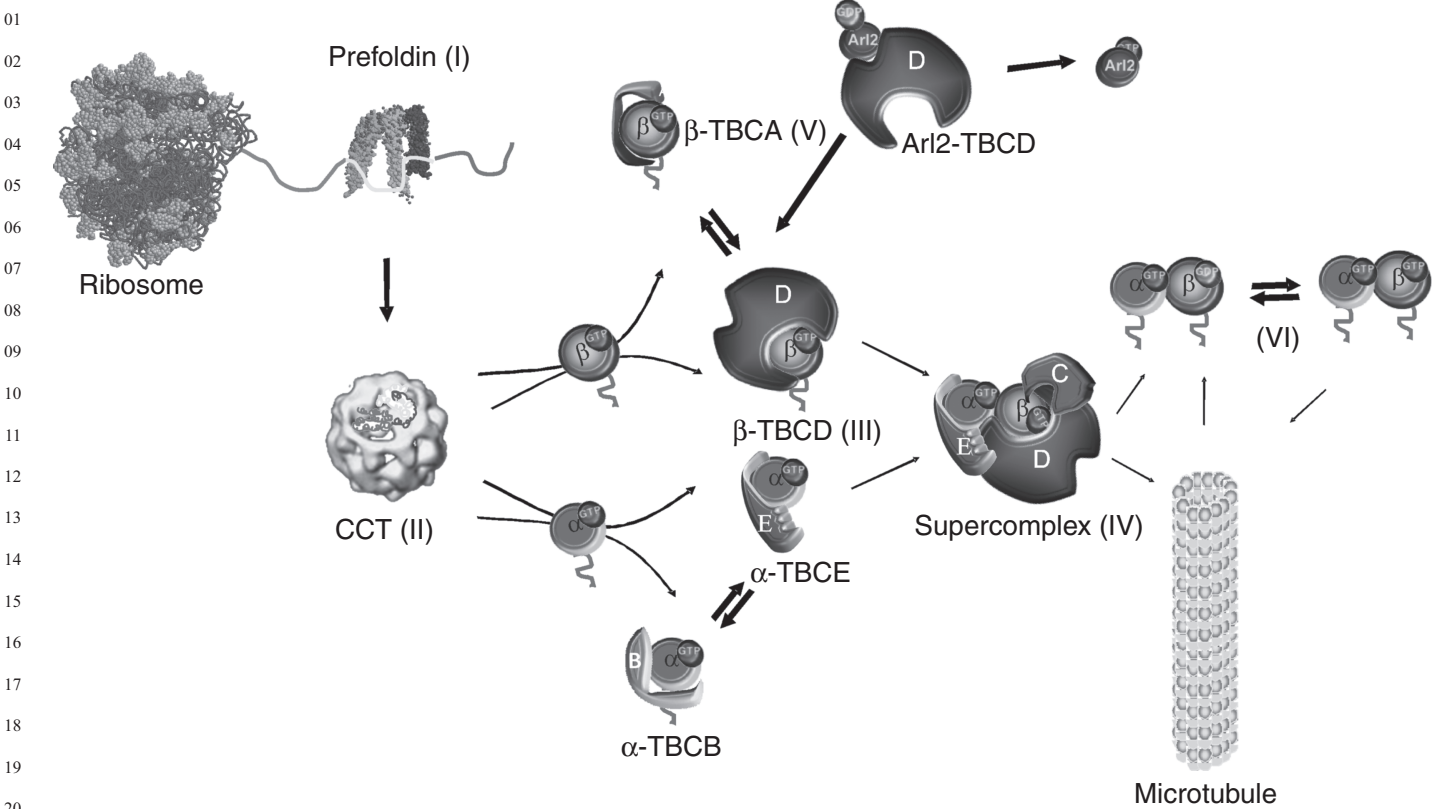

Fig. 1 Model of the tubulin-folding pathway in mammals adapted from Fanarraga et al. (2001, 2003). After synthesis, the tubulin polypeptides are protected by molecular chaperones that bind to prefoldin, which will next transfer these polypeptides to the cytosolic chaperonin chaperonin-containing Tcp-1. Five different tubulin-folding proteins, named tubulin cofactors, are then required in the post-chaperoninfolding pathway, which, after guanosine triphosphate hydrolysis, allows the release of the $\alpha \beta$-heterodimer. Although recent work suggests that several cofactors are involved in the proteasome pathway, the role of these cofactors in tubulin degradation is not known completely.

gel, we use a 75 - or 100 -ml Erlenmeyer flask. The polyacrylamide stock can be prepared in the laboratory, but it is more convenient to use a ready-to-use commercial solution, such as ProtoGel 30\% (w/v) acrylamide and $0.8 \%(\mathrm{w} / \mathrm{v})$ bisacrylamide from National Diagnostics, Hessle, England. The ProtoGel solution is mixed with 0.5 M MES, pH 6.7, $1 \mathrm{M} \mathrm{MgCl}_{2}$, and $0.5 \mathrm{M}$ ethylene glycol tetraacetic acid (EGTA) (Table I), and $10 \%(\mathrm{w} / \mathrm{v})$ ammonium persulfate and tetramethylethylenediamine (TEMED) are then added.

All reagents and solutions must be prepared with Milli-Q-purified water or equivalent. The recipe shown in Table I is for $40 \mathrm{ml}$ of solution, which is adequate for one gel measuring $14 \mathrm{~cm} \times 16 \mathrm{~cm}$ and $1.0 \mathrm{~mm}$ thick. Ten milliliters of solution is enough for two minigels measuring $7 \mathrm{~cm} \times 8 \mathrm{~cm}$ and $0.75 \mathrm{~mm}$ thick. Ammonium persulfate can be prepared and stored frozen in aliquots at $-20^{\circ} \mathrm{C}$, but we prefer to use it freshly made and we add TEMED just before polymerization. Pour the gel mix into the gel cast and insert the appropriate comb to allow the gel to polymerize for 30-60 min (Avila et al., 2008). When running a $14 \mathrm{~cm} \times 16 \mathrm{~cm}$ gel, we change the buffer in the cathode chamber every $2 \mathrm{~h}$. This technique can also be used to identify different polypeptides in the same complex by transferring the proteins from the native gel to nitrocellulose or a polyvinylidene fluoride membrane. Native protein transfer is performed as for conventional SDS gels, except that the native transfer 
Table I

Gel Preparation

\begin{tabular}{lcccc}
\hline Stock solution (ml) & \multicolumn{4}{c}{ Final acrylamide concentration in gel (\%) } \\
\cline { 2 - 5 } & 4 & 5 & 6 & 7 \\
\hline DW & 25.96 & 24.64 & 23.28 & 21.96 \\
$30 \%$ Acrylamide/0.8\% bisacrylamide & 5.32 & 6.64 & 8 & 9.32 \\
$0.5 \mathrm{M} \mathrm{MES} \mathrm{pH} 6.7^{\mathrm{M} \mathrm{MgCl}}$ & 8 & 8 & 8 & 8 \\
$0.5 \mathrm{MEGTA}^{a}$ & 0.04 & 0.04 & 0.04 & 0.04 \\
$\mathrm{M} \mathrm{GTP}^{a}$ & 0.08 & 0.08 & 0.08 & 0.08 \\
$10 \%(\mathrm{w} / \mathrm{v})$ APS & 0.04 & 0.04 & 0.04 & 0.04 \\
TEMED & 0.56 & 0.56 & 0.56 & 0.56 \\
& 0.04 & 0.04 & 0.04 & 0.04 \\
\hline
\end{tabular}

APS, ammonium persulfate; DW, distilled water; EGTA, ethylene glycol tetraacetic acid; GTP, guanosine triphosphate; TEMED, tetramethylethylenediamine.

${ }^{a}$ Inclusion of GTP in the gel mix and in the running buffer was shown to be essential for preservation of the native tubulin dimer as well as some of the complexes described in Fig. 1 (Zabala and Cowan, 1992).

buffer contains no methanol. Protein transfer requires the same duration and current conditions. After the gel is run, the bands of interest can be detected with immunodetection.

\section{B. Analysis of Purified Tubulin: Tubulin Stability Assessed Using Nondenaturing Gels}

Since the 1970s, many similar methods to purify microtubule proteins including tubulins and microtubule-associated proteins have been developed and reported (reviewed recently by Avila et al., 2008). Most protocols are based on the reversible assembly capacity of microtubule proteins. Although it is possible to polymerize microtubules in vitro in the presence of guanosine triphosphate (GTP) and at $35^{\circ} \mathrm{C}$, assembly-promoting agents such us glycerol or dimethyl sulfoxide (DMSO) are often included. The quality and quantity of tubulins and other microtubuleassociated proteins can be followed by SDS-PAGE analysis. However, this kind of electrophoresis does not tell us anything about the state of aggregation of these proteins and, thus, whether they are competent for microtubule assembly or interaction with other proteins. In other words, we cannot predict the amount of tubulin that becomes partially aggregated into intermediate polymerization structures and will not be competent for further experiments. In addition, we have observed that a single freezing step can cause the formation of some tubulin aggregates. To avoid these problems, we encourage the "polishing" of tubulin dimers through a gel filtration column. We routinely use Superdex-200, Superose-6, or Superose-12 columns (the column size depends on the amount to be purified), from Pharmacia GE Healthcare. GTP is often included in the column buffer for stabilization purposes, but we have found that this is not strictly required and may compromise further experiments.

Figure 2 shows a chromatogram of a gel filtration Superdex 200 PC 3.2/30 column. We used purified tubulin as described by Avila et al. (2008). After purification through the phosphocellulose column, tubulin is usually stored at $-80^{\circ} \mathrm{C}$. About $500 \mu \mathrm{g}$ of purified tubulin at $12 \mu \mathrm{g} / \mu \mathrm{l}$ is injected in the column, which has been 


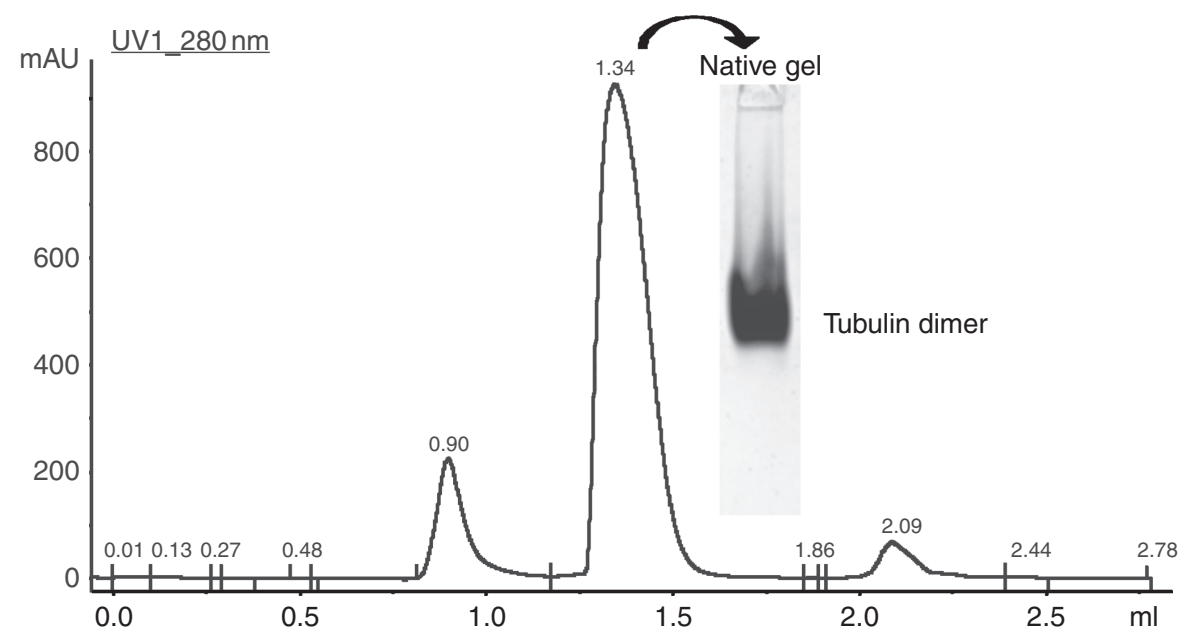

Fig. 2 Chromatographic elution profile obtained at $280 \mathrm{~nm}$ of absorbance from a gel filtration analysis of phosphocellulose-purified tubulin through a high-resolution Superdex 200 PC 3.2/30 column. Inset: nondenaturing gel ( $6 \%$ polyacrylamide) analysis of the peak corresponding to a molecular mass of $110 \mathrm{kDa}$ eluted from the column. The gel has been stained with Coomassie blue.

equilibrated with buffer A (0.1 MMES buffer, $\mathrm{pH} 6.7$, containing $1 \mathrm{mM} \mathrm{MgCl}_{2}$, $1 \mathrm{mMEGTA}$, and $25 \mathrm{mM} \mathrm{KCl}$ ). About $70-80 \%$ of the tubulin protein elutes as a homogeneous peak in the elution volume and corresponds to the size of the tubulin dimer. A proportion of tubulin may appear in the void volume of the column, suggesting the formation of large aggregates. The tubulin fractions containing dimers can be quantified using a NanoDrop spectrophotometer (Thermo Scientific, Wilmington, NC, USA) and kept on ice. About $15 \mu \mathrm{g}$ of protein in $10 \mu \mathrm{l}$ is then loaded onto a $6 \%$ nondenaturing minigel with native loading buffer (Avila et al., 2008, this work). As shown in the inset of Fig. 2, a large band corresponding to the tubulin dimer can be observed, whereas there is no staining at the origin of the gel, revealing that all the tubulin in the peak behaves as dimers that do not aggregate.

Because tubulin has long been considered a highly unstable protein, we wanted to further understand tubulin behavior at different temperatures and at different times and after thawing and refreezing many times in the presence of different reagents such as glycerol and DMSO used routinely as coadjuvants in polymerization experiments. Aliquots of $1.25 \mu \mathrm{g} / \mu \mathrm{l}$ tubulin were diluted with buffer A, frozen, and thawed $1,2,4,8$, or 16 times, as indicated in Fig. 3 , in the presence of $25 \%$ glycerol or $10 \%$ DMSO. These samples were frozen and thawed the same number of times before being loaded onto a $14 \mathrm{~cm} \times 16 \mathrm{~cm}$ nondenaturing gel. The results shown in Fig. 3 (panel B) show that, although the inclusion of glycerol preserved tubulin from denaturation or aggregation when frozen and thawed up to 16 times, $50 \%$ of the total tubulin in buffer A aggregated or unfolded after eight freeze-thaw cycles and $20 \%$ after only two cycles. The effect of freezing-thawing was greatest in the presence of DMSO. After 2 freeze-thaw cycles, the result was similar at the same dilution in buffer $\mathrm{A}$, but the effect was much greater after 16 cycles.

As mentioned above, it is believed that tubulin becomes unstable after several hours at $4^{\circ} \mathrm{C}$. To study the effect of temperature on the unfolding and aggregation of 
(A)

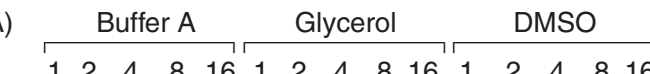

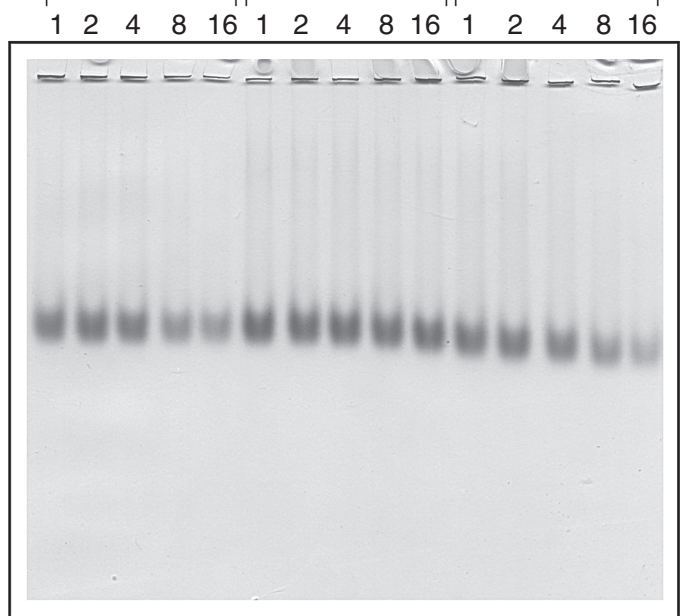

(B)

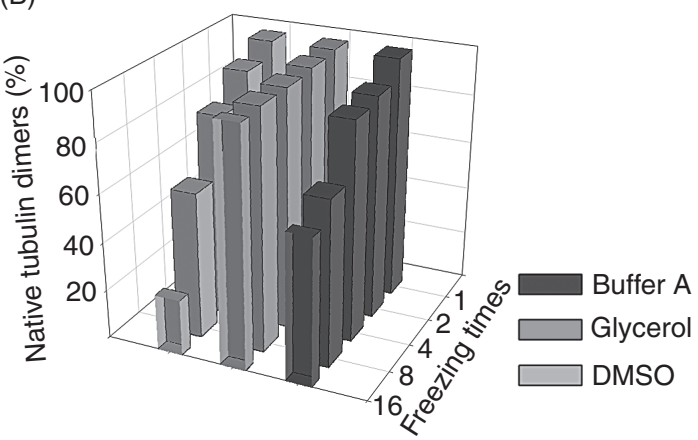

Fig. 3 (A) Nondenaturing polyacrylamide gel electrophoresis ( $6 \%$ polyacrylamide) of $4 \mu 1$-purified tubulin (at $1.25 \mu \mathrm{g} / \mu \mathrm{l}$ ) frozen for the times indicated in the presence of buffer, $25 \%$ glycerol, or $10 \%$ dimethyl sulfoxide. The gel was stained with Coomassie blue and scanned before drying. The bands were quantified using Image J $1.36 \mathrm{~b}$ (by Wayne Rasband, NIH, MD, USA) software freely available on the Internet. (B) Quantification of the percentage of tubulin heterodimers remaining. The threedimensional bar chart was made using SigmaPlot 8.0 software (Systat Software, Richmond, CA, USA).

the dimer, we incubated $1.25 \mu \mathrm{g} / \mu \mathrm{l}$ of purified tubulin, which had already been frozen once $(0 \mathrm{~h})$ at $4{ }^{\circ} \mathrm{C}$ and at $30^{\circ} \mathrm{C}$ for the times indicated in Fig. 4. Aliquots were then loaded onto a $14-\mathrm{cm} \times 16-\mathrm{cm}$ nondenaturing gel (panel A) and on a $14-\mathrm{cm} \times 16-\mathrm{cm}$ denaturing SDS gel (panel B). Although at both temperatures, tubulin dimers remained stable for $8 \mathrm{~h}$, the amount of tubulin dimer remaining active after $20 \mathrm{~h}$ of incubation declined to $65 \%$ at $4{ }^{\circ} \mathrm{C}$ and $30 \%$ at $30^{\circ} \mathrm{C}$ (panel C). After $30 \mathrm{~h}$, the percentage of folded tubulin was $44 \%$ at $4{ }^{\circ} \mathrm{C}$ and $7 \%$ at $30^{\circ} \mathrm{C}$. In addition, after $20 \mathrm{~h}$ at $30^{\circ} \mathrm{C}$, the stability of tubulin was compromised because of degradation (panel B). 
(A)
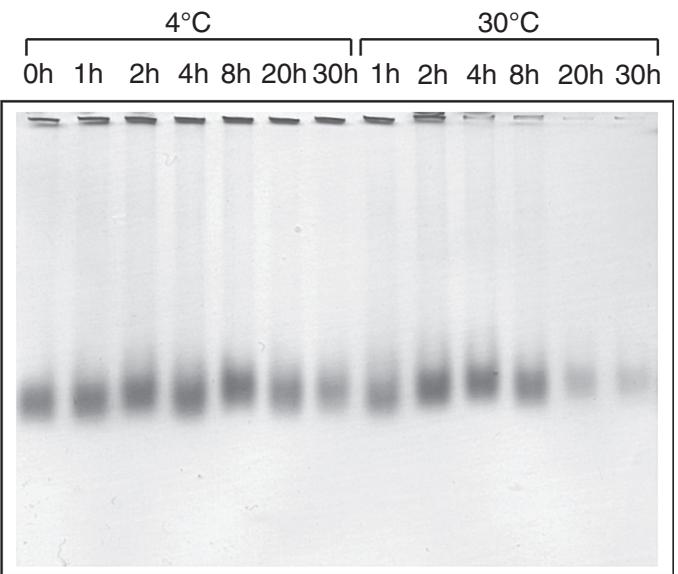

(B)

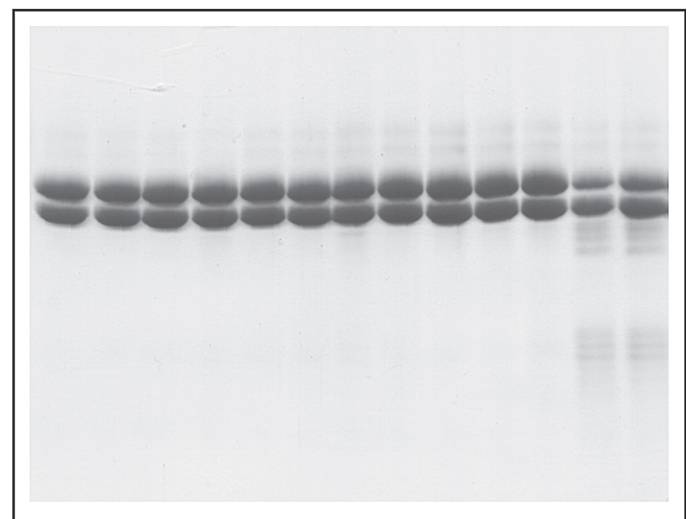

(C)

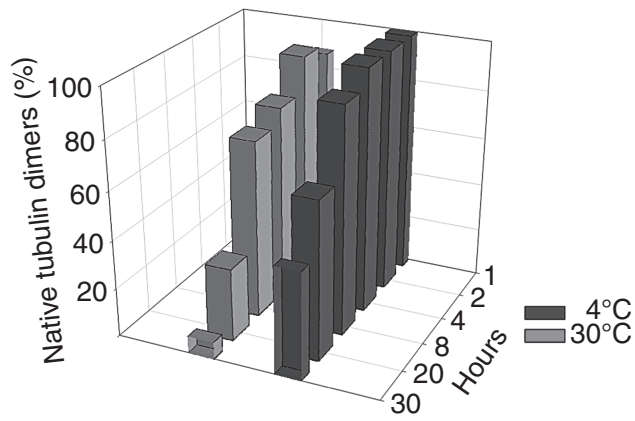

Fig. 4 Evaluation of the quality of native tubulin heterodimers by nondenaturing polyacrylamide gel electrophoresis (6\% polyacrylamide). (A) Characterization of $4 \mu 1$ aliquots of purified tubulin (at $1.25 \mu \mathrm{g} / \mu \mathrm{l}$ ) in $100 \mathrm{mMMES}$, pH 6.7, containing $25 \mathrm{mM} \mathrm{KCl}, 1 \mathrm{mM} \mathrm{MgCl}_{2}$, and $1 \mathrm{mMEGTA}$, incubated for the times indicated at 4 or $30^{\circ} \mathrm{C}$. Incubation time is indicated in hours. (B) The same tubulin samples were loaded onto an $8.5 \%$ sodium dodecyl sulfate gel. Both gels were stained with Coomassie blue and then scanned before drying. (C) Quantification of the percentage of tubulin heterodimers remaining. The three-dimensional bar chart was made using SigmaPlot 8.0 software (Systat Software, Richmond, CA). 


\section{Kinetic Analysis of Tubulin Complexes and Characterization of In Vitro-Synthesized Products}

The correct folding of tubulins and the generation of functional $\alpha \beta$-tubulin heterodimers require the participation of molecular chaperones such as prefoldin and chaperonin-containing Tcp-1 (CCT, Gao et al., 1993; Geissler et al., 1998; Vainberg et al., 1998) as well as several proteins known as tubulin-folding cofactors (A-E) (reviewed in Fanarraga et al., 2001, 2003; Fig. 1). Pulse-chase experiments can be used to follow the fate of newly synthesized tubulin during its folding and dimerization pathway. This system allows one to study tubulin-folding kinetics (Campo et al., 1994). Full-length cDNA encoding wild-type $\alpha_{6}$ - or $\beta_{3}$-tubulin cloned into appropriate vectors was used as the template and coupled to the in vitro transcription and translation (Craig et al., 1992) in the presence of ${ }^{35} \mathrm{~S}$-methionine for $20 \mathrm{~min}$ at $30^{\circ} \mathrm{C}$. Figure 5 shows the analysis of a 20 -min pulse with ${ }^{35} \mathrm{~S}$-methionine to allow the complete synthesis of $\alpha_{6}$-tubulin (panel A) and $\beta_{3}$-tubulin (panel B) isotypes. After the lag period (at $0 \mathrm{~min}$ ), synthesis of tubulin polypeptides was stopped with cold methionine and $1 \mathrm{mM}$ RNAse A $(0.08 \mu \mathrm{g} / \mu \mathrm{l})$. At the times indicated in Fig. 5, $2 \mu$ l aliquots of the in vitro reactions were frozen every
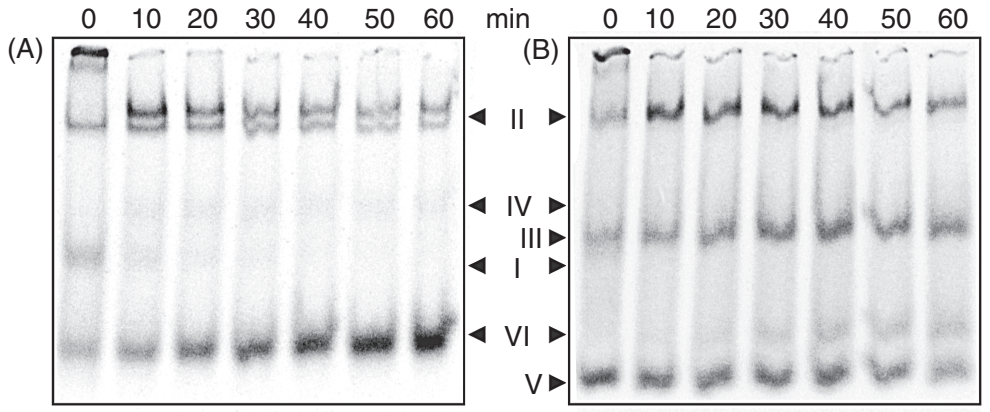

(C)

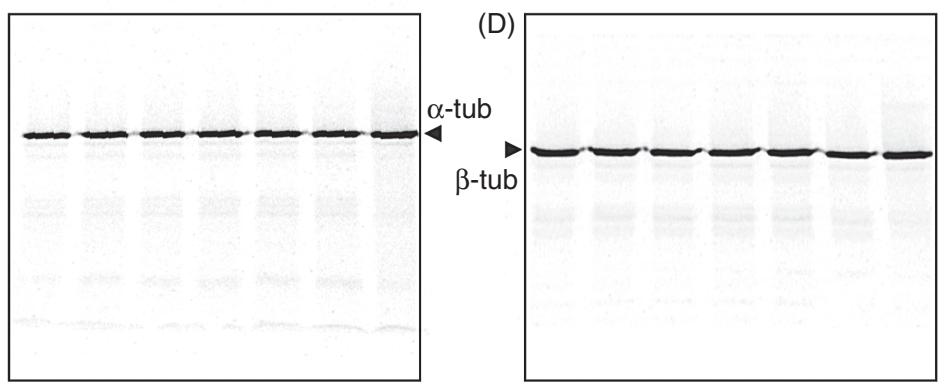

Fig. 5 Pulse-chase experiments of in vitro-translated tubulin isotypes. Newly synthesized $\alpha_{6}$-tubulin (A) and $\beta_{3}$-tubulin (B) isotypes were analyzed by electrophoresis on $4.5 \%$ nondenaturing gels. Panel A shows the results of a 20 min pulse that was chased with cold methionine and RNAse A and analyzed for the times indicated. Roman numerals indicate the different complexes that appeared during the in vitro translation of the tubulins depicted in Fig. 1 (see Section III). (C, D) The same tubulin samples run under denaturing conditions. The samples were diluted with the same volume of distilled water, $2 \times$ sodium dodecyl sulfate (SDS) loading buffer was added, and the samples were loaded onto an $8.5 \%$ SDSpolyacrylamide gel. Following electrophoresis, gels were fluorographed, dried, and exposed to film (Hyperfilm, GE Healthcare). 
10 min on dry ice. Samples were diluted with native loading buffer and loaded onto $6 \%$ native minigels.

As stated above, native electrophoresis allows one to identify the tubulin complexes as they are formed during the tubulin-folding process. This way, completely denatured or newly synthesized tubulin enters into the prefoldin complex (I) (Vainberg et al., 1998), which can be detected soon after initiation of synthesis. Prefoldin then transfers the tubulin substrate to CCT to form a binary complex (II), which appears as a band close to the origin (Fig. 5). This band containing the complex of CCT bound to tubulin was first shown to participate in tubulin biogenesis by Yaffe $e t$ al. (1992). For some $\beta$-tubulin isotypes such as $\beta_{3}$, this band, which has a molecular mass of about $900 \mathrm{kDa}$, precedes the two other bands corresponding to complexes (III) and (IV) (Fontalba et al., 1993; Tian et al., 1996). One band contains cofactor $\mathrm{D} / \beta$-tubulin complexes (lower band) and the other contains tubulin-folding cofactor E/ $\alpha$-tubulin in addition to cofactor D and $\beta$-tubulin (Fontalba et al., 1993; Tian et al., 1996). Finally, the two fast-migrating bands correspond to the $\alpha \beta$-tubulin heterodimers and the tubulin-folding cofactor A (TBCA)/ $\beta$-tubulin complexes (VI) and (V), respectively (Campo et al., 1994; Llosa et al., 1996).

\section{Isotype-Specific Antibody Analysis}

Tubulins are encoded by members of multigene families and are generally highly conserved at the sequence level except for the last 15 amino acids of their C-terminus, which are markedly divergent. The conserved peptide region in vertebrates constitutes the so-called isotype-defining region of tubulins. Because native electrophoresis allows the preservation of antibody-protein complexes, this technique can also be used to evaluate biochemically the affinity of an isotype-specific antibody. Protein-antibody complexes migrate differently during electrophoresis than do unbound protein monomers, resulting in retardation of the tubulin-containing band along the gel run, and the band retardation reflects the antibody binding. Further details on this technique and a more detailed study that evaluated the affinity of two polyclonal antibodies raised against the $\beta$-tubulin isotypes, $\beta 6$ and $\beta 2$, relative to all $\beta$-tubulin isotypes, has been published (Fanarraga et al., 1998). In this particular study, six different $\beta$-tubulin isotypes were translated in vitro using a reticulocyte cell-free translation system in the presence of ${ }^{35} \mathrm{~S}$-methionine (as explained above). The resulting reactions containing radiolabeled newly synthesized $\beta$-tubulin protein were next incubated with the two antibodies in two separated experiments, and the products of the incubation reactions were analyzed under nondenaturing gel electrophoresis where the various molecular forms of $\beta$-tubulin were separated and characterized. Dried gels were fluorographed and showed that, although the two polyclonal antibodies recognized the $\beta$-tubulin isotypes they were raised against, the anti- $\beta 2$ tubulin antibody appeared to be more specific than the anti- $\beta 6$-tubulin, which also recognized the platelet-specific $\beta 1$-tubulin isotype and resulted in a $38 \%$ reduction in radioactivity at the $\beta 1$-tubulin monomer band (Fanarraga et al., 1998).

\section{E. 2-D Native SDS Electrophoresis}

Folded $\alpha$ - and $\beta$-tubulins may exist as heterodimers in solution, forming microtubules or binding to the characterized tubulin-folding cofactors. Classical 2-D 
electrophoresis (2-D PAGE) allows one to separate the different polypeptides present in multimolecular complexes into individual species. The first dimension uses the isoelectric point as a differential characteristic to allow separation, and the second dimension uses the size under denaturing conditions in a SDS gel. This powerful method is used mainly to separate and identify peptides or proteins in complex samples for immunological or proteomic applications. A 2-D PAGE method using a native gel for the first dimension and a denaturing gel for the second dimension allows the separation of complexes under native conditions, and the composition can then be studied by SDS-PAGE. This technique also allows the determination of the stoichiometry of the different subunits in the complex. Here we show an example of an application of this technique to study the reactions involving tubulins synthesized in vitro; we used this method to study the formation of the different tubulin-folding intermediates described above.

The protein complexes are fractionated in the first dimension, and the second dimension is used to analyze the quality (length) and quantity of the in vitrosynthesized tubulin polypeptides (Fig. 6). These results demonstrate that tubulin polypeptides are constituents of the different complexes formed and allow us to exclude artifacts caused by the synthesis of partial products. The samples were loaded onto a native $0.75-\mathrm{mm}$ thick minigel $(7 \mathrm{~cm} \times 8 \mathrm{~cm})$ as described above. After $2 \mathrm{~h}$ of electrophoresis, a single running lane containing the electrophoresed sample was excised with a blade on glass, loaded onto a preparative $1.5 \mathrm{~mm}$ thick minigel $(7 \mathrm{~cm} \times 8 \mathrm{~cm})$, and fixed to the gel with $0.5 \%$ agarose prepared in $1 \times$ SDSloading buffer. Electrophoresis was performed for a further $2 \mathrm{~h}$ at $80 \mathrm{~V}$, after which the gel was dried and fluorographed as described above. As shown in Fig. 6, all bands contained full-length $\alpha_{6}$-tubulin (Fig. 6A and C) or $\beta_{3}$-tubulin (Fig. 6B and D) polypeptides. The Roman numerals indicate the position of the bands of the different tubulin complexes represented in Fig. 1 as detected in native gels.

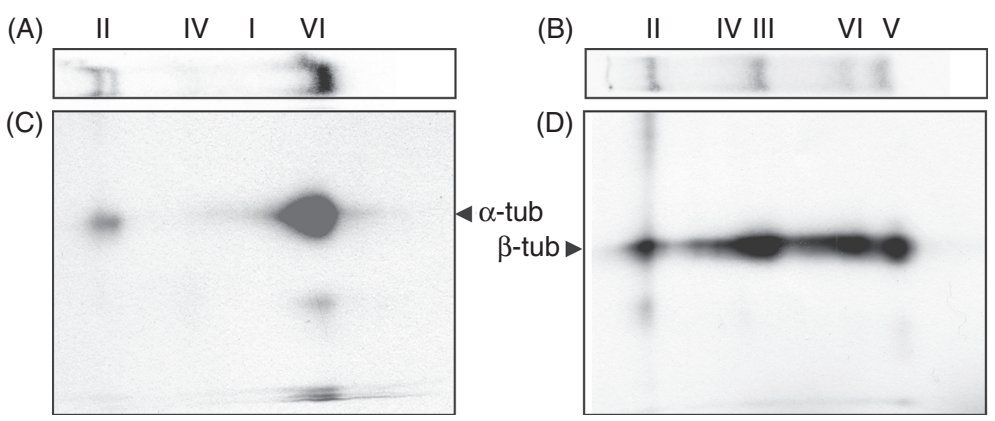

Fig. 6 Nonclassical two-dimensional native SDS-PAGE of in vitro-translated tubulin polypeptides. Aliquots of in vitro-translated $\alpha_{6^{-}}$and $\beta_{3^{-}}$-tubulin isotypes (A, B) incubated for $60 \mathrm{~min}$ at $30^{\circ} \mathrm{C}$ (Fig. 5) were loaded onto a $4.5 \%$ native minigel. Complete lanes containing this first dimension were excised from the gel and loaded onto an $8.5 \%$ sodium dodecyl sulfate polyacrylamide gel (C, D). After electrophoresis, the gels can be stained with Coomassie blue or fixed directly, and fluorographed. Panel $\mathrm{C}$ shows the second dimension for the $\alpha_{6}$-tubulin isotype and panel D shows the $\beta_{3}$-tubulin isotype. 


\section{F. Preparation and Analysis of Subtilisin-Treated Dimers}

Subtilisin cleaves tubulin subunits near the carboxy-terminus to generate two fragments. The larger fragment can coassemble into microtubules more efficiently than the whole molecule (Sacket et al., 1985; Serrano et al., 1984a,b). The small fragment corresponds to the highly acidic carboxy-terminus of tubulin needed for the interaction with mitogen-activated proteins and is believed to play a regulatory role in microtubule dynamics and tubulin folding (Fontalba et al., 1995; Littauer et al., 1986; Sacket et al., 1985; Serrano et al., 1984a,b; 1986). Subtilisin can be used to digest soluble tubulin or polymerized microtubules; the amount of enzyme can be manipulated to digest one or both tubulin subunits, or at different sites on the C-terminus. This technique was used by Hertzer and Walzak (2008) to study the influence of the C-terminus and the specific geometry of tubulin in the depolymerization activity of kinesin-13. Here, we describe a general protocol to eliminate the C-terminus (40-50 residues) of both subunits in solution. These tubulin heterodimers (named "S-dimers") can form microtubules and sheets in the presence of calcium ions (Lobert et al., 1993) and are useful for studying the contribution of the $\mathrm{C}$-terminus to the tubulin-folding, dimerization, and polymerization processes.

Limited proteolysis with subtilisin was performed essentially as described by Serrano et al. (1984a,b). Tubulin $(4 \mathrm{mg} / \mathrm{ml})$ was incubated at $25^{\circ} \mathrm{C}$ in $50 \mathrm{mM} \mathrm{MES}$, $\mathrm{pH}$ 6.7, containing $1 \mathrm{mMEGTA}$ and $0.5 \mathrm{mMMgCl}_{2}$ with different amounts of subtilisin (w/w) for $30 \mathrm{~min}$ as shown in Fig. 7A. After subtilisin digestion, the reaction was cooled to $4^{\circ} \mathrm{C}$ and stopped with $1 \mathrm{mM}$ phenylmethylsulfonyl fluoride (PMSF) made freshly in DMSO and $1 \mu \mathrm{M}$ aprotinin. Tubulin was repolymerized once in the presence of $1 \mathrm{mMGTP}$ and $2 \mathrm{mM} \mathrm{CaCl}_{2}$ for $30 \mathrm{~min}$ at $35^{\circ} \mathrm{C}$ and then

(A)

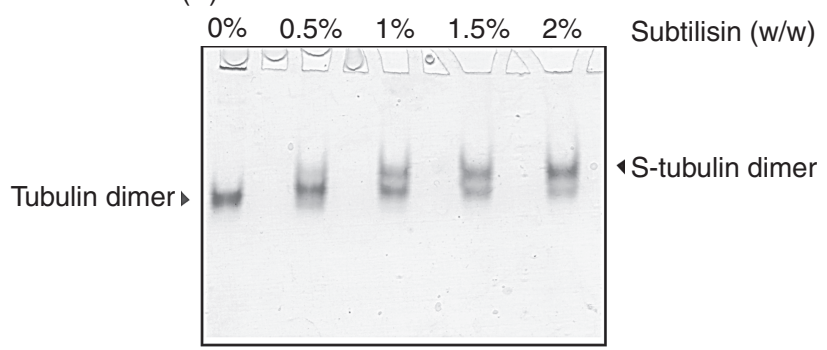

(B)

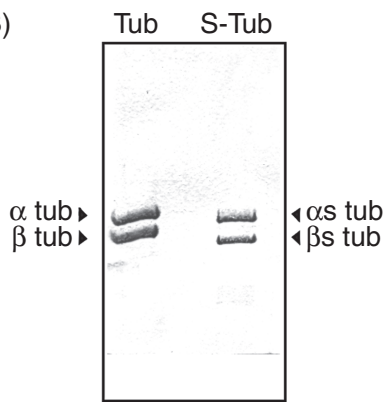

Fig. 7 (A) Monitoring the progress and efficiency of the subtilisin digestion of purified tubulin dimers using a nondenaturing polyacrylamide gel and sodium dodecyl sulfate polyacrylamide gel electrophoresis. (B) Symbols indicate the position of cow brain native tubulin dimers $(\alpha \beta)$ and subtilisin-digested dimers $\left(\alpha_{s} \beta_{s}\right)$. 
loaded with a Pasteur pipette on a prewarmed sucrose cushion in a tube. The microtubules made of S-tubulin were next pelleted at $100,000 \times g$ for $90 \mathrm{~min}$ at $35^{\circ} \mathrm{C}$ in a Beckman TLX centrifuge and then resuspended in $100 \mathrm{mMMES}, \mathrm{pH}$ 6.7, containing $0.3 \mathrm{M} \mathrm{KCl}$ and $1 \mathrm{mM} \mathrm{MgCl} 2$ at $4^{\circ} \mathrm{C}$ for $30 \mathrm{~min}$ to allow depolymerization. Insoluble material was removed by ultracentrifugation at $100,000 \times g$ for $20 \mathrm{~min}$ at $4^{\circ} \mathrm{C}$. Following this procedure, the different digestion reaction products were studied by electrophoresis under native conditions (as shown in Fig. 7A) to monitor the progress and efficiency of the experiment. For this purpose, aliquots diluted to onefifth with $25 \%$ sucrose (final concentration of $5 \%$ ) made up in $50 \mathrm{mMMES}, \mathrm{pH} 6.7$, were loaded onto a $6 \%$ nondenaturing polyacrylamide gel (Avila et al., 2008, this work; Zabala and Cowan, 1992). In parallel, an aliquot of S-tubulin was also analyzed in an $8.5 \%$ SDS gel (Fig. 7B) to check that the C-terminal region of both subunits had been digested by the enzyme and that there was no contamination with native undigested tubulin after the polymerization cycle in the presence of calcium ions.

Upon electrophoresis, untreated purified brain tubulin dimers gave a single band when analyzed by electrophoresis under nondenaturing conditions (Fontalba $e t$ al., 1995; Zabala and Cowan, 1992). This differed from the S-tubulin dimers, which also gave a single band (Fig. 7: $\alpha_{\mathrm{s}} \beta_{\mathrm{s}}$ ) that migrated more slowly than the corresponding wild-type tubulin heterodimer band. This difference reflects the greater negative net charge at $\mathrm{pH} 6.7$ of the untreated tubulin heterodimer compared with the same protein after removal of the carboxy-terminal domain.

\section{G. Other Applications of Nondenaturing Electrophoresis: Stability Study of the In Vivo Tubulin Complexes by Native Electrophoresis}

Mammalian tubulin complexes can also be examined by nondenaturing gel electrophoresis of soluble protein extracts. In this experiment, we intended to test the stability of TBCA $/ \beta$-tubulin dimer in soluble protein extracts at $4{ }^{\circ} \mathrm{C}$ obtained from $\mathrm{HeLa}$ cells, a standard tumor cell line, which were cultured in monolayers in a $5 \% \mathrm{CO}_{2}$-humidified atmosphere at $37^{\circ} \mathrm{C}$ in Dulbecco's modified Eagle's medium containing GlutaMAX (Invitrogen) supplemented with 10\% fetal calf serum (Invitrogen) and nonessential amino acids (Invitrogen). A nearly confluent 100-mm plate of HeLa cells was rinsed twice with $5 \mathrm{ml}$ of PBS and the cells were scraped into the last $5 \mathrm{ml}$ of PBS and centrifuged at $2500 \mathrm{rpm}$ for $3 \mathrm{~min}$ in a bench centrifuge. The cell pellet was then resuspended in $100 \mu \mathrm{l}$ of lysis buffer (0.1 MMES, pH 6.7, containing $1 \mathrm{mMMgCl}_{2}, 1 \mathrm{mMEGTA}, 0.1 \mathrm{mMGTP}, 5 \mathrm{nM}$ paclitaxel, $0.2 \mathrm{mM}$ dithiothreitol, $0.1 \mathrm{mM}$ PMSF, and protease inhibitor cocktail) and then lysed using a 2-ml Dounce homogenizer (20 strokes with a tight pestle). All procedures were performed at room temperature to avoid microtubule depolymerization because cold shock increases the pool of soluble tubulin heterodimers and would thus affect the amount and composition of tubulin complexes.

The cellular lysate obtained was centrifuged at $14,000 \times g$ for $30 \mathrm{~min}$ at $20^{\circ} \mathrm{C}$ in a standard bench centrifuge, and the supernatants were recovered (soluble fraction). We used native electrophoresis to investigate the stability of the TBCA/ $\beta$-tubulin complex (Fig. 1, complex (V)) under different conditions. We compared $30 \mu \mathrm{g}$ of total soluble protein fractions freshly obtained with the same extract after being kept at $4^{\circ} \mathrm{C}$ for 48 or $96 \mathrm{~h}$. Electrophoresis was performed in a native $1.5-\mathrm{mm}$ thick minigel $(7 \mathrm{~cm} \times 8 \mathrm{~cm})$ for $2 \mathrm{~h}$ at $80 \mathrm{~V}$, after which the proteins were transferred to a 


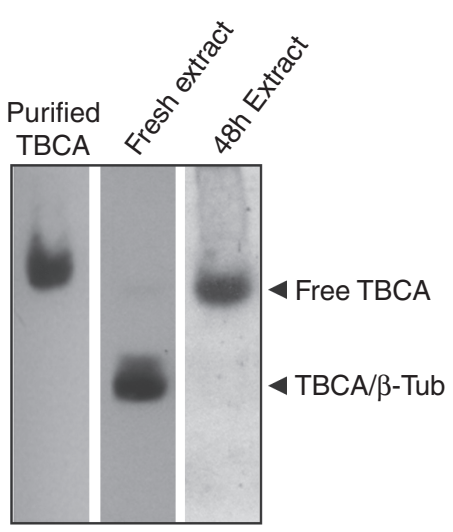

Fig. 8 Analysis of the tubulin-folding cofactor A (TBCA) $/ \beta$-tubulin complex stability at $4^{\circ} \mathrm{C}$. A total of $30 \mu \mathrm{g}$ of freshly obtained soluble protein extract prepared from HeLa cells was compared with the same extract after $48 \mathrm{~h}$ storage at $4{ }^{\circ} \mathrm{C}$ using nondenaturing gel electrophoresis $(6 \%)$ and TBCA detection by Western blotting. This study reveals that the TBCA $/ \beta$-tubulin complex disassembles after storage.

nitrocellulose membrane for Western blotting. Immunodetection was performed using a rabbit polyclonal antiserum against TBCA (1:5000), which has been characterized broadly (Llosa et al., 1996). Figure 8 shows clearly that the TBCA/ $\beta$ tubulin complex was present in the fresh extract but absent in extracts stored at $4{ }^{\circ} \mathrm{C}$ for $48 \mathrm{~h}$, indicating that this complex is not stable under these conditions.

\section{Materials}

- Gel solutions (Table I)

- Protein sample to be analyzed

- $4 \times$ loading buffer

- Electrophoresis equipment (we use Mini-PROTEAN from Bio-Rad and 0.75-mm spacers or sturdier vertical slab units from Amersham Biosciences, GE Healthcare)

- 0.5 M MES pH 6.7 (53.3 g MES monohydrate dissolved in $400 \mathrm{ml}$ water, titrated to $\mathrm{pH} 6.7$ with $\mathrm{KOH}$ or $\mathrm{NaOH}$, and adjusted to a final volume of $500 \mathrm{ml}$ )

- $1 \mathrm{M} \mathrm{MgCl}_{2}$

- 0.5 M EGTA [19 g EGTA-ethylene glycol-bis-(2-aminoethyl)- $N, N, N^{\prime}, N^{\prime}$ tetraacetic acid dissolved in $80 \mathrm{ml}$ water, titrated to $\mathrm{pH} 8$ with $\mathrm{KOH}$ or $\mathrm{NaOH}$, and adjusted to a final volume of $100 \mathrm{ml}]$

- Electrophoresis (running) buffer (0.1 M MES-KOH or MES-NaOH, pH 6.7, $1 \mathrm{mM} \mathrm{MgCl}_{2}, 1 \mathrm{mM}$ EGTA, diluted in $700 \mathrm{ml}$ water, $160 \mathrm{ml} 0.5 \mathrm{M}$ MES, $800 \mu \mathrm{l}$ $1 \mathrm{M} \mathrm{MgCl}_{2}$, and $1.6 \mathrm{ml} \mathrm{0.5} \mathrm{MEGTA,} \mathrm{and} \mathrm{adjusted} \mathrm{to} \mathrm{a} \mathrm{final} \mathrm{volume} \mathrm{of} 800 \mathrm{ml}$ )

- $10 \times$ transfer buffer $[15.13 \mathrm{~g}$ Tris $(250 \mathrm{mM})$ and $72.067 \mathrm{~g}$ glycine $(1.92 \mathrm{M})$ dissolved in pure water and adjusted to a final volume of $500 \mathrm{mls}$ ]

- $1 \mathrm{M} \mathrm{GTP}\left(1.5 \mathrm{ml}\right.$ distilled $\left.\mathrm{H}_{2} \mathrm{O}, 1 \mathrm{~g} \mathrm{Na}_{2} \mathrm{GTP}\right)$; bring to final volume of $1.91 \mathrm{ml}$ with $\mathrm{H}_{2} \mathrm{O}$; do not allow to stand at room temperature or at $4^{\circ} \mathrm{C}$, but aliquot in small volumes and store at $-70^{\circ} \mathrm{C}$ immediately 
- Rabbit reticulocyte lysates from Promega

- ${ }^{35} \mathrm{~S}$-methionine $(>1000 \mathrm{Ci} / \mathrm{mmol})$ from Amersham Biosciences, GE Healthcare

- Antibodies against the protein of interest; in this work, we raise antibodies to tubulin cofactors in our laboratory

\section{Discussion}

A main problem in denaturing gel electrophoresis is that it cannot be used to study protein-protein interactions. These interactions can occur in specific cell locations or at a specific time, and studying these interactions could contribute to understanding further the cellular processes involving tubulin. We have reviewed the use of a nondenaturing electrophoresis technique complemented with the well-known SDS denaturing electrophoresis. The various applications of this technique mentioned could complement gel filtration analysis to identify different types of protein complexes formed during the tubulin-folding and tubulin-assembly process. This technique allows one to determine the stability of some of the complexes that participate in tubulin biogenesis and has been used extensively in the study of the folding process of actin and other proteins that also require specific molecular chaperones. We also show how native electrophoresis is useful for the study of tubulin complexes that are present inside the cell before purification. This would allow one to study these complexes inside the cell or a tissue under different conditions and would represent an important tool to study the physiologic roles of tubulin and other proteins.

\section{Summary}

One of the main purposes of this technique is to provide an easy way to investigate tubulin complexes in a way that is relevant both physiologically (i.e., complexes with other proteins) and biochemically (i.e., protein aggregates). Among others, we have presented results to show how to proceed when we want to know if a tubulin solution is competent for polymerization studies or for interaction studies with proteins of interest. We have also shown how this type of electrophoresis can be used to study the electrophoretic changes resulting from the removal of the carboxyl terminus of this protein, how a particular tubulin complex disassembles with time, and how one might take advantage of the properties of this system to investigate the quality of isotype specificity of a determined antibody. We believe native electrophoresis is a fundamental tool for modern proteomic studies.

\section{Acknowledgments}

This work was supported by grants from the Consolider-Ingenio Spanish Ministry of Education and Science (Centrosome-3D and BFU2007-64882) and the Instituto de Formación e Investigación Marques de Valdecilla (IFIMAV). We also thank Begoña Ubilla for technical help. 


\section{References}

Avila J., Soares H., Fanarraga M. L., and Zabala J. C. (2008). Isolation of microtubules and microtubule proteins. Curr. Protoc. Cell Biol. Chapter 3, Unit 3.29.

Burré J, Wittig I., and Schägger H. (2009). Non-classical 2-D electrophoresis. Methods Mol. Biol. 564, 33-57.

Campo R, Fontalba A, Sanchez L. M., and Zabala J. C. (1994). A $14 \mathrm{kDa}$ release factor is involved in GTP-dependent $\beta$-tubulin folding. FEBS Lett. 353, 162-166.

Craig D., Howell M. T., Gibbs C. L. Hunt T., and Jackson R. J. (1992). Plasmid cDNA-directed-protein synthesis in a coupled transcription-translation system. Nucleic Acids Res. 20, 4987-4995.

Davis, B. J. (1964). Disc electrophoresis. II. Method and application to human serum proteins. Ann. N. Y. Acad. Sci. 121, 404-427.

Fanarraga M. L., Aloria K., Avila J., and Zabala J. C. (1998). Characterization of tubulin isotype-specific antibodies by electrophoretic mobility shift assay. Biotechniques 25, 940-942.

Fanarraga M. L., Avila J., Guasch A., Coll M., and Zabala J. C. (2001). Post-chaperonin tubulin folding cofactors and their role in Microtubule dynamics. J. Struct. Biol. 135, 219-229.

Fanarraga, M. L., Abad X., Kortazar D., Bellido J., Villegas J. C., and Zabala J. C. (2003). Structure and function of the mammalian tubulin folding cofactors. In "Recent Research Developments in Biochemistry" (S. G., Pandalai, ed.) Vol. 4, pp. 575-587. Research Signpost, Trivandrum - 695023, Kerala, India.

Fontalba, A., Avila, J., and Zabala, J. C. (1995). $\beta$-tubulin folding is modulated by the isotype-specific carboxy-terminal domain. J. Mol Biol. 246, 628-636.

Gao, Y., Vainberg, I. E., Chow, R. L., and Cowan, N. J. (1993). Two cofactors and cytoplasmic chaperonin are required for the folding of $\alpha$ - and $\beta$-tubulin. Mol. Cell. Biol. 13, 2478-2485.

Geissler, S., Siegers, K. and Schiebel, E. (1998). A novel protein complex promoting formation of functional alpha- and gamma-tubulin. EMBO J. 17, 952-966.

Hertzer, K. M., and Walczak, C. E. (2008). The C-termini of tubulin and the specific geometry of tubulin substrates influence the depolymerization activity of MCAK. Cell Cycle 7, 2727-2737.

Laemmli, U. K. (1970). Cleavage of structural proteins during the assembly of the head of bacteriophage T4. Nature 227, 680-685.

Littauer, U. Z., Giveon, D., Thierauf, M., Ginzburg, I. and Ponstingl, H. (1986). Common and distinct tubulin binding sites for microtubule-associated proteins. Proc. Natl. Acad. Sci. U. S.A. 83, 7162-7166.

Llosa, M., Aloria, K., Campo, R., Padilla, R., Avila, J., Sanchez-Pulido, L. and Zabala, J. C. (1996). The $\beta$-tubulin monomer release factor (p14) has homology with a region of the DnaJ protein. FEBS Lett. 397, 283-289.

Lobert, S, Hennington, B. S. and Correia, J. J. (1993). Multiple sites for subtilisin cleavage of tubulin: effects of divalent cations. Cell Motil. Cytoskel. 25, 282-297.

Markert, C. L. and Hunter, R. L. (1959). The distribution of esterases in mouse tissues. J. Histochem. Cytochem. 7, 42-49.

Ornstein, L. (1964). Disc electrophoresis. I. Background and theory. Ann. N. Y. Acad. Sci. 121, 321-349.

Safer, D. (1994). Nondenaturing polyacrylamide gel electrophoresis NPAGE as a method for studying protein interactions. In "Cell Biology: A Laboratory Handbook" (J. E. Celis, ed.) Vol. 3, pp. 218-221. Academic Press, Inc., San Diego, CA.

Sackett, D. L., Bhattacharyya, B. and Wolff, J. (1985). Tubulin subunit carboxyl termini determine polymerization efficiency. J. Biol. Chem. 260, 43-45.

Serrano, L., Avila, J., and Maccioni, R. B. (1984a). Controlled proteolysis of tubulin by subtilisin: localization of the site for MAP2 interaction. Biochemistry 23, 4675-4681.

Serrano, L., de la Torre, J., Maccioni, R. B., and Avila, J. (1984b). Involvement of the carboxyl-terminal domain of tubulin in the regulation of its assembly. Proc. Natl. Acad. Sci. U.S.A. 81, 5989-5993.

Shapiro, A. L., Vinuela, E., and Maizel, J. V., Jr. (1967). Molecular weight estimation of polypeptide chains by electrophoresis in SDS-polyacrylamide gels. Biochem. Biophys. Res. Commun. 28, 815-820.

Smithies, O. (1955). Zone electrophoresis in starch gels: Group variations in the serum proteins of normal human adults. Biochem. J. 61, 629-641.

Tian, G., Huang, Y., Romelaere, H., Vanderkerckhove, J., Ampe, C., and Cowan, N. J. (1996). Pathway leading to correctly folded $\beta$-tubulin. Cell 86, 287-296. 
Vainberg, I. E., Lewis, S. A., Rommelaere, H., Ampe, C., Vandekerckhove, J., Klein, H. L., and Cowan, N. J. (1998). Prefoldin, a chaperone that delivers unfolded proteins to cytosolic chaperonin. Cell 93, 863-873.

Weber, K., and Osborn, M. (1969). The reliability of molecular weight determinations by dodecyl sulfatepolyacrylamide gel electrophoresis. J. Biol. Chem. 244, 4406-4412.

Wittig, I., and Schägger, H. (2008). Features and applications of blue-native and clear-native electrophoresis. Proteomics 8, 3974-3990.

Yaffe, M. B., Farr, G. W., Miklos, D., Horwich, A. L., Sternlicht, M. L., and Sternlicht, H. (1992). TCP1 complex is a molecular chaperone in tubulin biogenesis. Nature. 358, 245-248.

Zabala, J. C., and Cowan, N. J. (1992). Tubulin dimer formation via the release of alpha- and beta-tubulin monomers from multimolecular complexes. Cell Motil. Cytoskel. 3, 222-230. 
Chapter No: 5

\section{Query No Contents}

Please note that "DW" in "Table I" has been expanded as "distilled water". Please check.

Please provide the zip code for the first affiliation.

Please provide the manufacturer location for "Pharmacia GE Healthcare" in the sentence "We routinely use ..."

"Fontalba et al. (1993)" is cited in the text but not provided in the reference list. Please check.

Please note that "MAPs" has been changed to "mitogen-activated proteins" in the sentence "The small fragment ...". "Serrano et al. (1986)" is not listed in the reference list. Please confirm.

Please provide the manufacturer location for "Invitrogen" in the sentence "In this experiment, ..."

Please note that as per style unit "rpm" is allowed, so please change the unit to " $g$ " in all occurrences.

Please provide the manufacturer location for "Bio-Rad" in the sentence "Electrophoresis equipment ..."

10 please provide the manufacturer location for "Promega" in the sentence "Rabbit reticulocyte lysates" 